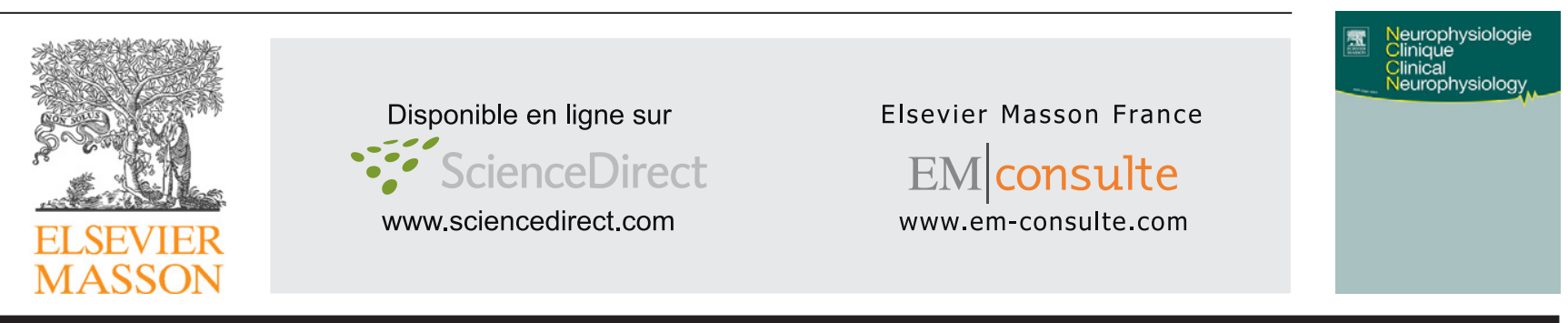

ORIGINAL ARTICLE/ARTICLE ORIGINAL

\title{
Personality modulation of P300 wave recorded within an emotional oddball protocol
}

\section{Modulation par la personnalité de l'onde P300 enregistrée dans un protocole oddball émotionnel}

\section{S. Mardaga*, M. Hansenne}

\author{
Personality and Individual Differences Unit, University of Liège, boulevard du Rectorat 5, bat B32, 4000 Liege, Belgium
}

Received 24 September 2008; accepted 27 December 2008

Available online 24 January 2009

\section{KEYWORDS \\ Personality; \\ Cloninger; \\ Emotion; \\ Event-related potentials; \\ P300}

\section{MOTS CLÉS}

Personnalité ;

Cloninger ;

Émotions ;

Potentiels évoqués

liés à l'événement ;

P300

\begin{abstract}
Summary
Objectives. - Several studies reported that personality modulates responses to emotional stimuli, including cognitive and attentional aspects of the emotional response. The aim of this study was to refine these results while using visual event-related potentials (ERPs) and referring to Cloninger's personality model.

Methods. - ERPs were recorded in 46 normal subjects within a visual oddball protocol with checkerboards as the standard stimuli and pictures selected as neutral, pleasant or unpleasant from the International Affective Picture System as the target stimuli.

Results. - N200 amplitude was smaller and P300 amplitude was larger following the presentation of pleasant pictures in low-harm avoidance but not high-harm avoidance subjects.

Conclusions. - These results support the idea that both automatic and selective cognitive processing of emotional pictures is modulated by personality.

(c) 2009 Elsevier Masson SAS. All rights reserved.
\end{abstract}

\section{Résumé}

Buts. - De nombreuses études ont rapporté que la personnalité module les réponses émotionnelles, notamment l'aspect cognitif et attentionnel des réponses émotionnelles. Le but de la présente étude est d'affiner ces résultats en utilisant des potentiels visuels liés à l'événement et en faisant référence au modèle de personnalité décrit par Cloninger.

Méthode. - Les potentiels évoqués ont été enregistrés chez 46 sujets sains lors d'un protocole oddball visuel utilisant des damiers comme stimuli standard et des images neutres, plaisantes et déplaisantes tirées de l'International Affective Picture System comme images-cibles.

Résultats. - L'amplitude de la N200 était plus faible et l'amplitude de l'onde P300 plus importante après la présentation d'images plaisantes chez les sujets présentant un score bas à l'évitement du danger mais pas chez les sujets caractérisés par un score élevé à cette dimension.

\footnotetext{
* Corresponding author.

E-mail address: solange.mardaga@student.ulg.ac.be (S. Mardaga).
}

0987-7053/\$ - see front matter @ 2009 Elsevier Masson SAS. All rights reserved. 
Conclusions. - Ces résultats supportent l'idée que les traitements automatiques et sélectifs des stimulations émotionnelles sont modulés par la personnalité.

(c) 2009 Elsevier Masson SAS. Tous droits réservés.

\section{Introduction}

A large body of research demonstrated that personality influences emotional responses. Indeed, extraversion and the Behavioral Activation System (BAS) were related to positive emotions, while neuroticism and the Behavioral Inhibition System (BIS) were related to negative affects $[6,14,25,28,30]$. More particularly, the cognitive aspects of emotion were shown to be involved in this relationship $[4,24,42]$. Thus, Rusting [42] reported positive correlations between extraversion and the generation of homophones and story completion with positive content, and the free recall of positive words, while neuroticism was correlated to the generation of homophones and story completion with negative content, and the free recall of negative words. These results suggest that neuroticism and extraversion would modulate the cognitive processing of unpleasant and pleasant information, respectively.

Event-related potentials (ERP) are a critical way to address several cognitive processes (e.g., attentional processes, memory and language) in normal as well as pathological subjects [21]. P300 is certainly one of the most studied ERP. It is elicited when a subject detects an unexpectable stimulus and consists of two components labeled $\mathrm{P} 3 \mathrm{a}$ and $\mathrm{P} 3 \mathrm{~b}$. The P3a (or novelty $\mathrm{P} 3$ ) is a frontocentral wave that reflects an aspect of the orienting response and has been related to evaluative attentional processes [23,31,39]. The P3b is a centroparietal wave that has been linked to the task-relevant and decision-related character of the eliciting stimulus. It reflects memory updating process $[31,39]$ or processing closure [49] and might indicate the transfer of relevant information to consciousness [37].

Several previous studies reported that P300 amplitude depends (among other parameters) on personal relevance of the information, including its emotional aspect $[1,19,35]$. Thus, using a three-stimulus visual oddball protocol with emotional pictures as the novel stimulus, Delplanque et al. [19] reported an increase in P300 amplitude for pleasant and unpleasant novel stimuli relative to neutral novel ones, which indicates that attentional processes indexed by P300 are influenced by emotional valence and arousal characteristics of information.

Considering that personality dimensions modulate emotional reactivity, several studies investigated personality influence on P300s elicited by emotional stimuli. Most of these studies focused on the P3a while recording ERPs within single-stimulus protocols or following decision-unrelated stimuli $[2,15,16]$; these studies generally reported no differential influence of personality dimensions (extraversion and neuroticism) on P300 amplitude following pleasant and unpleasant stimuli. Conversely, within an oddball protocol, De Pascalis et al. [17] recently reported that anxious (high-BIS) subjects showed larger P3b amplitude and impulsive (high-BAS) subjects disclosed smaller P3b amplitude to unpleasant target words, when compared to non-anxious and low-impulsivity subjects, respectively. This suggests that memory updating processes are modulated by personality, which is in keeping with previously-reported correlations between BIS-BAS and the processing of emotional information. Moreover, recent studies conducted within non-clinical samples showed that subclinical forms of psychopathy and anxiety modulate the processing of emotional stimuli (namely faces), as indexed by ERPs, and the authors suggested that these modulations might be due to personality differences [5,41].

In this study, personality will be addressed in reference to Cloninger's biosocial model of personality, which assumes four innate temperaments (novelty seeking [NS], harm avoidance [HA], reward dependence and persistence) and three acquired characters (self-directedness, cooperativeness and self-transcendence) $[7,8]$. Briefly, NS is the tendency to respond actively to novel stimuli leading to pursuit of rewards and escape from punishment. HA is the tendency to inhibit responses to signals of aversive stimuli that leads to avoidance of punishment and non-reward. Reward dependence is the tendency for a positive response to conditioned signals of reward that maintains behaviour. Persistence is perseverance despite frustration and fatigue. Self-directedness is the ability of an individual to control, regulate and adapt his or her behaviour to fit the situation in accord with individually chosen goals and values. Cooperativeness accounts for individual differences in identification with and acceptance of other people. Self-transcendence is associated with spirituality. More particularly, NS was defined as a tendency to respond intensely to indices of potential reward and $\mathrm{HA}$ as a tendency to respond intensely to cues for aversive stimuli. Moreover, NS and HA originally refer to the BAS and BIS, respectively [7]. Therefore, highNS subjects were expected to show particular sensitivity to pleasant pictures, whereas high-HA subjects were expected to show preferential sensitivity to unpleasant pictures.

The aim of the present study is thus to replicate previous findings that personality modulates cognitive processing of emotional stimuli, refining those results to selective attention and memory updating processes indexed by $\mathrm{P} 3 \mathrm{~b}$, on the one hand, and extending them to personality dimensions as described by Cloninger's biosocial model, on the other hand.

\section{Methods}

\section{Subjects}

The experiment was conducted on 46 participants ( 23 males) aged between 20 and 27 years (mean $=22.8, S D=1.7)$. All participants were naïve to the aim of the experiment and to the pictures used in the protocol. All had normal or corrected-to-normal vision. The ethical committee of the 
University of Liège Psychology Faculty approved the protocol and the participants gave their informed consent to take part to the study.

Participants filled out the French version of the 240 -items self-questionnaire Temperaments and Characters Inventory, revised (TCl-R) [9], translated by Pélissolo et al. [36]).

\section{Visual stimuli}

The P300 was recorded using a visual oddball protocol with a white and red checkerboard as the standard stimulus and emotional pictures as target stimuli. Emotional pictures consisted of 75 coloured pictures selected as neutral, pleasant and unpleasant ( 25 in each category) from the International Affective Picture System (IAPS [33]) ${ }^{1}$. The slides were displayed on a 17 -inch computer screen $(1400 * 1050$ pixels, rate of refreshment $60 \mathrm{~Hz}$ ) at a one meter distance from the subject.

\section{Procedure}

On arrival, subjects completed the TCI-R and the electrodes were attached. Recording sessions were then run, they lasted about 11 minutes. Subjects were instructed to press a key as quickly as possible whenever a target picture appeared. After the recording session, subjects were presented the same pictures once again and asked to rate pleasure and emotional arousal for each one. Rating scales for picture pleasure and arousal consisted of 9-point (1-9) Likert scales, ranging from "unpleasant" to "pleasant" and from "calm" to "arousing", respectively.

\section{ERP recordings and data reduction}

ERP recording, stimulus presentation and waveform analyses were performed with an ANT system (eeprobe, eevoke and eemagine EEG respectively). ERP recordings were conducted while the subjects were comfortably seated in a sound-attenuated room. ERPs were elicited using a visual oddball protocol with $80 \%$ standard stimuli (checkerboards) and $20 \%$ deviant stimuli (emotional pictures), presented in a pseudo-randomized order, so that at least two standard pictures preceded a target one. A total of 375 pictures (75 emotional pictures and 300 checkerboards) was displayed during 1 second each, with inter-stimulus-interval lasting $850 \pm 50 \mathrm{~ms}$.

EEG was recorded at 12 sites of the 10-20 System (Fz, F3, F4, Cz, C3, C4, Pz, P3, P4, Oz, 01, O2), using $\mathrm{Ag} / \mathrm{AgCl}$ electrodes, earlobes for reference and forehead for ground. All sites were abraded before recording to maintain an

\footnotetext{
${ }^{1}$ Neutral pictures: 1121, 1302, 1321, 1390, 1560, 1603, 1811, 1942, 2000, 2160, 2220, 5470, 5629, 5920, 5940, 5950, 7640, 8030, $8041,8190,8200,8370,9190,9411,9582$; pleasant pictures: 1463, $1710,1722,1920,2050,2170,2208,2209,2303,2352,2550,2620$, 4607, 4510, 4611, 4651, 4659, 4660, 4666, 4670, 4680, 8470, 8501; for women: 4460, 4520; for men: 4220, 4290; unpleasant pictures: $1200,2141,3071,3110,3181,3230,3550,6020,6230,6570,6831$, $6940,8230,9090,9102,9120,9230,9402,9433,9560,9561,9570$, 9630, $9810,9910$.
}

impedance below $5 \mathrm{~K} \Omega$. Amplifier gains were set at 20, with a band pass of $0.05-35 \mathrm{~Hz}$. The EEG was recorded during $900 \mathrm{~ms}$ from stimulus onset, with a $150 \mathrm{~ms}$ pre-stimulus baseline. Trials on which the EEG or EOG exceeded 30 standard deviations of amplitude-distribution were rejected automatically [48]. Trials on which participants had not correctly pressed the key were rejected too. There was no other rejection criterion and individual curves were not systematically inspected visually for artefacts.

P100, N100, P200, N200 and P300 were scored as the maximum positive and negative peaks between 70-160, 90-200, $150-250,220-320$, and 300-600 ms, respectively.

\section{Statistical analysis}

Statistical analyses were conducted with Statistica (7.1) for Windows. Friedman Anovas with picture category (neutral, positive and negative) as a repeated-measure factor were conducted with pleasure and arousal ratings as the dependent variables; Wilcoxon test were used for paired comparisons. Pearson's correlations were conducted between all personality dimensions from the $\mathrm{TCl}-\mathrm{R}$. In order to investigate interactions between electrophysiological and personality data, median splits were applied according to subjects' scores on NS and HA dimensions, and subjects were assigned to high- or low-NS group and to highor low-HA group. A three-way Anova $2 \mathrm{NS}$ groups $\times 2 \mathrm{HA}$ groups $\times 3$ picture categories was performed with reaction times for dependent variable. Mean amplitudes and latencies of each scored waves were analyzed with four-way repeated-measure Anovas 2 NS groups $\times 2$ HA groups $\times 3$ picture categories (neutral, positive and negative) $\times 12$ electrode locations (Fz, F3, F4, Cz, C3, C4, Pz, P3, P4, Oz, $01,02)$, with picture category and electrode location as the repeated-measure factors.

For all Anovas, the Greenhouse-Geisser correction was applied to significance levels for effects including a repeated-measure factor and Student's t tests were used as post-hoc analysis.

\section{Results}

Friedman Anovas (3 picture categories) revealed significant effects with both pleasure $\left(\chi_{(2)}^{2}=87.75, p<0.0001\right)$ and arousal $\left(\chi_{(2)}^{2}=45.48, p<0.0001\right)$ as the dependent variables, with significant differences between all picture categories. Mean pleasure for neutral, positive and negative pictures was 4.9, 6.2 and 2.6 respectively and mean arousal 4.7, 5.2 and 5.9. Descriptive characteristics of the groups are reported in Table 1 and Pearson's correlations between personality dimensions are depicted in Table 2.

The Anova performed with reaction times as the dependent variable revealed no main effect of picture category $\left(F_{(2,76)}=1.39, p=0.26\right)$. A significant NS $\times \mathrm{HA}$ interaction was revealed $\left(F_{(1,38)}=4.69, p=0.04\right)$, showing that, regardless of the picture category, high-HA subjects responded slower than low-HA ones, within low-NS $\left(\mathrm{t}_{(38)}=4.69, p=0.04\right)$ only. No interaction between personality dimensions and picture category reached significance with reaction times as the dependent variable. 
Table 1 Subject number, mean age and sex repartition in low-NS and high-NS and low-HA and high-HA groups.

\begin{tabular}{llccc}
\hline & Low-NS & High-NS & Low-HA & High-HA \\
\hline N (male/female) & $23(12 / 11)$ & $23(11 / 12)$ & $22(12 / 10)$ & $20(8 / 12)$ \\
Age & 23.2 & 22.5 & 22.6 & 22.9 \\
NS score & 92.6 & 119.0 & 109.6 & 100.6 \\
HA score & 97.1 & 88.3 & 80 & 106.8 \\
\hline
\end{tabular}

NS: novelty seeking; HA: harm avoidance.

Table 2 Mean, median and SD for all personality dimensions assessed and Pearson's correlations between personality dimensions (correlations in bold are significant at $p<0.05$ threshold).

\begin{tabular}{|c|c|c|c|c|c|c|c|}
\hline & NS & $\mathrm{HA}$ & $\mathrm{RD}$ & $P$ & SD & $\mathrm{C}$ & ST \\
\hline Mean & 105.83 & 92.70 & 109.33 & 113.22 & 137.15 & 139.28 & 69.46 \\
\hline Median & 106.5 & 92 & 111 & 112.5 & 136.5 & 141 & 68 \\
\hline SD & 17.61 & 15.31 & 12.01 & 18.41 & 18.10 & 13.79 & 15.28 \\
\hline NS & 1.00 & -0.46 & 0.42 & -0.15 & 0.11 & 0.01 & 0.18 \\
\hline $\mathrm{HA}$ & - & 1.00 & 0.02 & -0.36 & -0.35 & -0.07 & -0.21 \\
\hline $\mathrm{RD}$ & - & - & 1.00 & -0.09 & 0.08 & 0.19 & 0.10 \\
\hline$P$ & - & - & - & 1.00 & 0.51 & 0.08 & -0.05 \\
\hline SD & - & - & - & - & 1.00 & 0.46 & -0.21 \\
\hline$C$ & - & - & - & - & - & 1.00 & 0.10 \\
\hline
\end{tabular}

NS: novelty seeking; HA: harm avoidance; RD: reward dependency; P: persistence; SD: self-directedness; C: cooperation; ST: selftranscendence.

Grand average waves recorded while subjects were presented standard checkerboards, and target neutral, pleasant, and unpleasant pictures are depicted in Fig. 1. The effects of electrode and picture category from the four-way repeated-measure Anovas conducted with P100, $\mathrm{N} 100, \mathrm{P} 200, \mathrm{~N} 200$ and P300 amplitudes and latencies as the dependent variables are presented in Table 3 . The analyses performed with wave amplitudes as the dependent variables reflected that unpleasant pictures evoked smaller P100 than neutral pictures at posterior sites $(02, \mathrm{P} 2)$, that pleasant pictures evoked larger P200 than neutral and unpleasant pictures at frontal and central sites but smaller P200 at occipital sites, that pleasant pictures elicited smaller N200 than neutral and unpleasant pictures at frontal and central sites, and that pleasant pictures elicited larger P300 than neutral and unpleasant ones at frontal and central sites. The analyses performed with peak latencies as the dependent variables reflected that P100 was faster after the presentation of pleasant pictures than neutral ones and that $\mathrm{N} 200$ was faster after the presentation
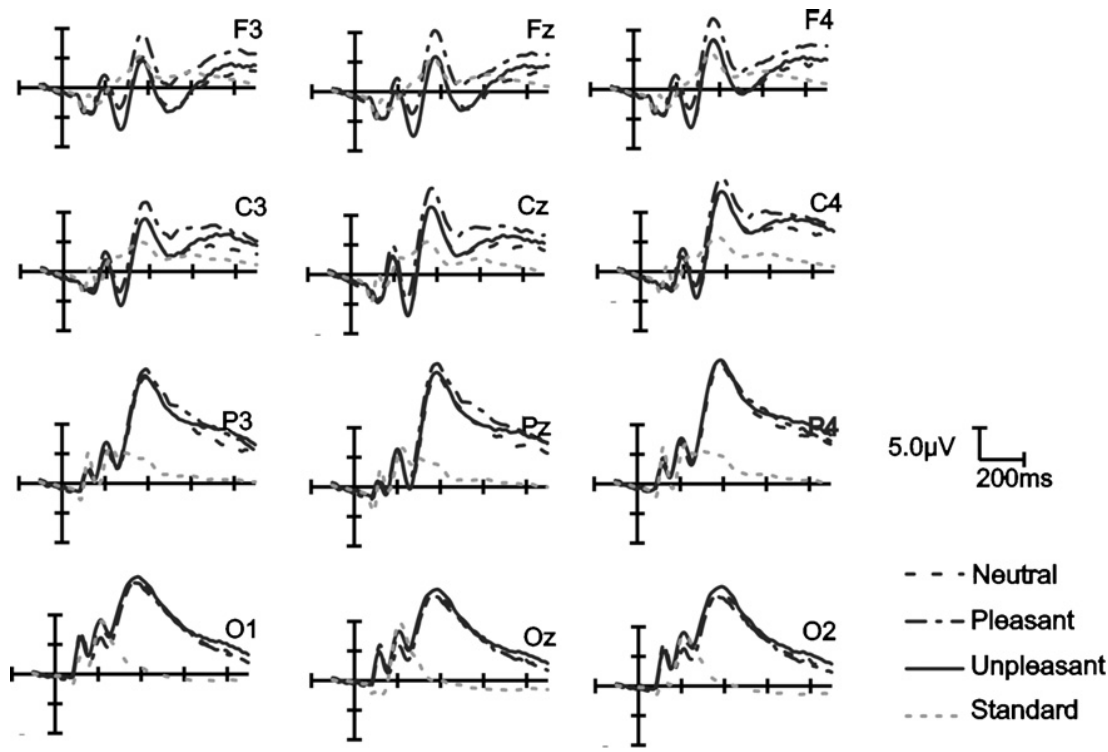

Figure 1 Grand average waves recorded while subjects were presented standard checkerboards and target neutral, pleasant and unpleasant pictures. 
Table 3 F-values and their probabilities for Anovas performed with P100, N100, P200, N200 and P300 amplitudes and latencies as the dependent variables.

\begin{tabular}{|c|c|c|c|c|}
\hline \multicolumn{2}{|c|}{ Dependent variable } & \multirow{2}{*}{$\begin{array}{l}\text { Effect } \\
\text { Electrode }\end{array}$} & \multirow{2}{*}{$\begin{array}{l}F(d f) \\
73.52(11,418)\end{array}$} & \multirow{2}{*}{$\frac{p}{<0.0001}$} \\
\hline P100 & Amplitude & & & \\
\hline & & Picture category & $0.16(2,76)$ & 0.85 \\
\hline & & Picture category $\times$ electrode & $2.73(22,836)$ & 0.02 \\
\hline & Latency & Electrode & $8.06(11,418)$ & $<0.0005$ \\
\hline & & Picture category & $7.99(2,76)$ & $<0.001$ \\
\hline & & Picture category $\times$ electrode & $2.05(22,836)$ & 0.03 \\
\hline \multirow[t]{6}{*}{ N100 } & Amplitude & Electrode & $27.84(11,418)$ & $<0.0001$ \\
\hline & & Picture category & $0.16(2,76)$ & 0.81 \\
\hline & & Picture category $\times$ electrode & $1.88(22,836)$ & 0.07 \\
\hline & Latency & Electrode & $10.80(11,418)$ & $<0.0001$ \\
\hline & & Picture category & $2.47(2,76)$ & 0.09 \\
\hline & & Picture category $\times$ electrode & $1.92(22,836)$ & 0.05 \\
\hline \multirow[t]{6}{*}{ P200 } & Amplitude & Electrode & $25.40(11,418)$ & $<0.0001$ \\
\hline & & Picture category & $0.86(2,76)$ & 0.42 \\
\hline & & Picture category $\times$ electrode & $25.06(22,836)$ & $<0.0001$ \\
\hline & Latency & Electrode & $10.71(11,418)$ & $<0.0001$ \\
\hline & & Picture category & $0.17(2,76)$ & 0.84 \\
\hline & & Picture category $\times$ electrode & $0.78(22,836)$ & 0.64 \\
\hline \multirow[t]{6}{*}{ N200 } & Amplitude & Electrode & $59.16(11,418)$ & $<0.0001$ \\
\hline & & Picture category & $5.78(2,76)$ & 0.005 \\
\hline & & Picture category $\times$ electrode & $19.09(22,836)$ & $<0.0001$ \\
\hline & Latency & Electrode & $9.57(11,418)$ & $<0.0001$ \\
\hline & & Picture category & $11.02(2,76)$ & $<0.0001$ \\
\hline & & Picture category $\times$ electrode & $0.81(22,836)$ & 0.61 \\
\hline \multirow[t]{6}{*}{ P300 } & Amplitude & Electrode & $44.35(11,418)$ & $<0.0001$ \\
\hline & & Picture category & $4.48(2,76)$ & 0.02 \\
\hline & & Picture category $\times$ electrode & $15.58(22,836)$ & $<0.0001$ \\
\hline & Latency & Electrode & $6.38(11,418)$ & 0.002 \\
\hline & & Picture category & $0.07(2,76)$ & 0.91 \\
\hline & & Picture category $\times$ electrode & $0.93(22,836)$ & 0.50 \\
\hline
\end{tabular}

of pleasant pictures relative to neutral or unpleasant ones.

A significant $\mathrm{HA} \times$ electrode $\times$ valence $\left(\mathrm{F}_{(22,836)}=2.34\right.$, $p=0.01$ ) was shown with P200 latency as the dependent variable, reflecting that in low-HA subjects, P200 was faster after the presentation of pleasant pictures than unpleasant ones, at central site $\left(\mathrm{C} 3 ; \mathrm{t}_{(38)}=2.24, p=0.03\right)$. Finally, a significant NS $\times$ HA interaction was also revealed with N200 latency as the dependant variable $\left(F_{(1,38)}=5.16, p=0.03\right)$, reflecting that high-HA subjects show generally slower N200 than low-HA ones $\left(\mathrm{t}_{(38)}=2.16, p=0.04\right)$, within low-NS subject group only. Regarding P100, N100 and P200, no other main or interaction effect of personality dimensions reached significance, with amplitudes as well as latencies as the dependent variable.

Regarding N200 amplitudes, a significant $\mathrm{HA} \times$ picture category interaction with N200 amplitude as the dependent variable was revealed $\left(\mathrm{F}_{(2,76)}=4.27, p=0.02\right)$. The paired comparisons revealed that pleasant pictures elicited smaller N200 than neutral or unpleasant ones in low-HA subjects $\left(\mathrm{t}_{(38)}=4.08, p<0.001\right.$ and $\left.\mathrm{t}_{(38)}=3.93, p<0.001\right)$, whereas no difference was found within high-HA subjects $\left(\mathrm{t}_{(38)}=0.74\right.$, $p=0.46$ and $\mathrm{t}_{(38)}=0.01, p=0.99$ ) (see Fig. 2).
Regarding P300 amplitudes, a marginal $\mathrm{HA} \times$ picture category interaction with P300 amplitude as the dependent variable was revealed $\left(F_{(2,76)}=3.02, p=0.06\right)$. Paired comparisons showed that pleasant pictures elicited larger

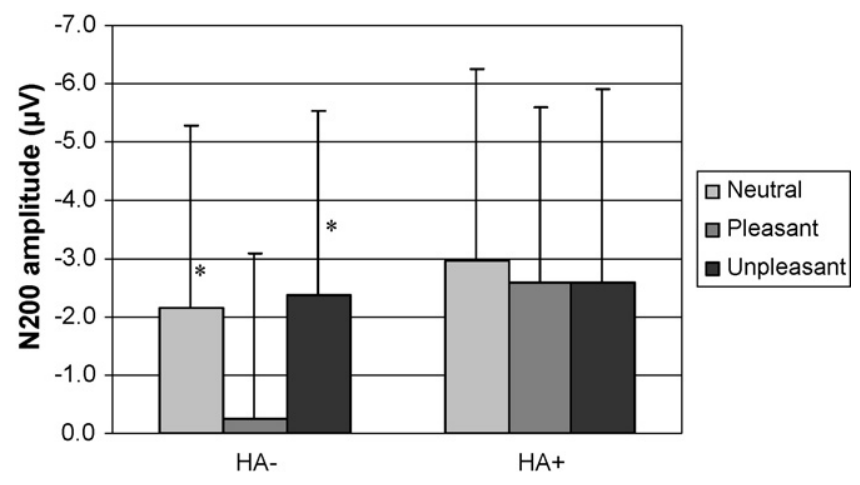

Figure 2 Mean (SEM) N200 amplitude (all electrodes) elicited by neutral, pleasant and unpleasant pictures in low-HA (HA-) and high-HA $(\mathrm{HA}+)$ subjects. * indicates a significant difference at $p<0.001$ relative to pleasant pictures. 


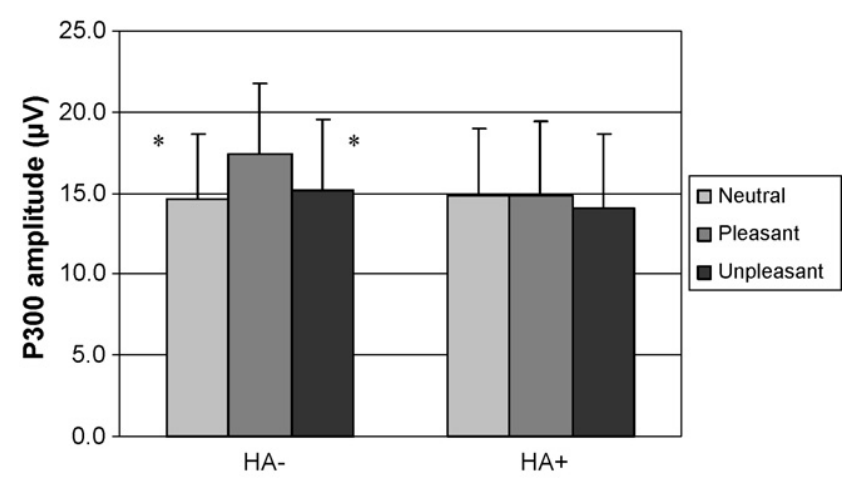

Figure 3 Mean (SEM) P300 amplitude (all electrodes) elicited by neutral, pleasant and unpleasant pictures in low-HA (HA-) and high-HA $(\mathrm{HA}+)$ subjects. * indicates a significant difference at $p<0.05$ relative to pleasant pictures.

P300 than neutral or unpleasant ones in low-HA subjects $\left(\mathrm{t}_{(38)}=4.08, p<0.001\right.$ and $\left.\mathrm{t}_{(38)}=2.84, p<0.01\right)$, whereas no difference was found within high-HA subjects $\left(\mathrm{t}_{(40)}=0.06\right.$, $p=0.95$ and $t_{(40)}=0.91, p=0.37$ ) (see Fig. 3).

A significant $\mathrm{HA} \times$ picture category $\times$ electrode interaction was revealed with $\mathrm{P} 300$ latencies as the dependent variable $\left(F_{(22,836)}=2.03, p=0.03\right)$. Paired comparisons showed that P300 latency was longer at parietal site (P1) following pleasant pictures than neutral ones in high- $\mathrm{HA}\left(\mathrm{t}_{(38)}=2.56\right.$, $p=0.01$ ) but not in low-HA subjects $\left(\mathrm{t}_{(38)}=0.38, p=0.70\right)$. Neither main nor interaction effect of other personality dimensions reached significance.

\section{Discussion}

This study aimed to examine the influence of personality on emotional information processing while recording visual ERPs. More particularly, it specifically focused on P3b component, using an emotional oddball paradigm, thus clarifying previous findings on selective attention and decisional processes. Moreover, it extends previous findings to NS and HA dimensions from Cloninger's biosocial model.

In keeping with previous studies [1,18,19], emotional (pleasant, in the present study) pictures elicited larger $\mathrm{P} 300$ s relative to neutral ones. Moreover, the amplitudes of earlier components (P100, P200, N200, but not N100) were also modulated by the emotional character of visual stimuli, which is also in keeping with previous studies [3,19,32,38]. These results suggest that early processing, as well as both automatic and selective attentional allocation to emotional stimuli relative to neutral ones, might be favoured, which is consistent with the large body of literature that emphasizes the major importance of pre-attentional processes in emotional responses $[34,40]$. In particular, the decrease in N200 amplitude to pleasant pictures might reflect the fact that pleasant pictures are perceived as a cue of low danger, as previously suggested [46], and therefore require less automatic attention than unpleasant or neutral pictures. On the other hand, pleasant pictures elicited larger P300, indicating that they involved more selective attention than neutral or unpleasant pictures.

The reason why unpleasant pictures did not elicit more automatic or selective attention than neutral ones is unclear and contrasts with some studies [19,32]. As a possible explanation, the rated arousal parameters of both pleasant and unpleasant pictures were rather close to the neutral ones (even if the difference was statistically significant), which might suggest that low-arousal unpleasant pictures would not require particular attention, whereas low-arousal pleasant pictures, as they are closer to ecological pleasant stimuli, would elicit attention modulation. Thus, both valence and arousal parameters of the presented stimuli would influence attentional allocation.

Regarding personality-emotion interaction, the major finding of our study is that low-HA subjects showed smaller N200 amplitudes and larger P300 following pleasant pictures relative to neutral and unpleasant ones, whereas high-HA subjects did not. The fact that high-HA subjects did not show any N200 decrease following pleasant pictures, whereas low-HA did, might be a sign of their previously described tendency to maintain a high level of attentional resources toward the environment [12,29], even within a pleasant context [13]. This would constitute a part of a constant defensive attitude typically observed in high-HA subjects, described as anxious and danger anticipating [8]. Conversely, N200 amplitude was not differentially increased as a function of personality dimensions. Thus, personality dimensions would not differentially favour automatic orienting response toward emotional stimuli (here indexed by N200). This is consistent with the general absence of modulation by personality of $\mathrm{P} 3 \mathrm{a}$ amplitude recorded after the presentation of emotional stimuli $[2,15,16]$.

The increased P3b amplitude following pleasant pictures in low-HA subjects only suggests a particular sensitivity to cues of potential reward in these subjects. This modulation of P3b amplitude following emotional pictures presentation by personality is consistent with the recent result reported by De Pascalis et al. [17] that high-BIS subjects showed larger P3b amplitudes than low-BIS ones and high-BAS subjects showed smaller P3b amplitudes than low-BAS ones, following the presentation of unpleasant target words. More particularly, our results give some support to the joint system hypothesis $[10,11]$, which states that both BAS- and BIS-related personality dimensions modulate sensitivity to pleasant and unpleasant stimulations: high-BIS and low-BAS subjects would be the most reactive to unpleasant pictures, while low-BIS and high-BAS subjects would be the most reactive to pleasant pictures. So, not only BAS would increase positive affect sensitivity, but also BIS would decrease it. This result suggests that high-HA subjects would be to some extent barred from focusing attention on pleasant pictures, which may explain that anxious subject are classically found to remind fewer pleasant stimuli [26] or report less positive affects $[20,47]$. The fact that only HA dimension was found to modulate sensitivity to pleasant pictures in this study could suggest that, contrarily to its theoretical definition, NS dimension may not be a good measure of BAS sensitivity. Indeed, NS is a measure close to impulsivity [22] and although impulsivity was classically associated to BAS sensitivity, several studies recently reported that, in spite of possible shared variance, impulsivity is a distinct component of personality and therefore an unsatisfactory (or incomplete) predictor of reward sensitivity [22,43,44].

Finally, why reactivity to unpleasant pictures was not modulated by personality deserves some discussion. As 
already mentioned, the unpleasant pictures that were used in this study have a somehow low emotional arousal level and, therefore, might not closely fit ecological unpleasant stimuli and not elicit significant negative affects. This is supported by the result that neither N200 nor P300 amplitude was increased following unpleasant pictures relative to neutral ones. Another possibility is that HA (as well as other BIS measures) is only a partial measure of defence-system sensitivity, as recent papers emphasized the difference between BIS (i.e. anxiety system, close to HA dimension) and FightFlight-Freezing System (FFFS, i.e. panic system) [27,45]. Though both BIS and FFFS are parts of the defence system, FFFS is the properly reacting to potential punishments unit, whereas BIS mostly manages contradictions between conflicting goals [27]. Therefore, although the previously advanced relations between negative affects sensitivity and BIS seem to remain generally relevant $[10,11]$, exclusively BIS-related personality dimensions might be incomplete predictors of punishment sensitivity.

In summary, our results support the idea that some personality traits modulate emotional reactivity to different valence stimulations and that pre-attentive cognitive processes as well as selective attention might be involved in this modulation. More particularly, a low-HA score might be predictive of less automatic attentional allocation but more selective attention to pleasant stimuli.

\section{Conflicts of Interest}

None.

\section{Acknowledgements}

We thank Thomas Colemans, Laurence Galère and François Valkenborgh for their technical support.

\section{References}

[1] Amrhein C, Mühlberger A, Pauli P, Wiedemann G. Modulation of event-related brain potentials during affective picture processing: a complement to startle reflex and skin conductance response? Int J Psychophysiol 2004;54:231-40.

[2] Bartussek D, Becker G, Diedrich O, Naumann E, Maier S. Extraversion, neuroticism and event-related brain potentials in response to emotional stimuli. Pers Individ Diff 1996;20:301-12.

[3] Batty M, Taylor MJ. Early processing of six basic facial emotional expression. Cogn Brain Res 2003;17:613-20.

[4] Bradley BP, Mogg K. Mood and personality in recall of positive and negative information. Behav Res Ther 1994;32:137-41.

[5] Campanella S, Vanhoolandt ME, Philippot P. Emotional deficit in subjects with psychopathic tendencies as assessed by the Minnesota Multiphasic Personality Inventory-2: an event-related potentials study. Neurosci Lett 2005;373:26-31.

[6] Canli T, Sivers H, Withfield SL, Gotlib IH, Gabrieli JDE. Amygdala response to happy faces as a function of extraversion. Science 2002;296:2191.

[7] Cloninger CR. A systematic method for clinical description and classification of personality variants. Arch Gen Psychiatry 1987; 44:573-88.

[8] Cloninger CR, Svrakic DM, Przybeck TR. A psychobiological model of temperaments and character. Arch Gen Psychiatry 1993;50:975-90.
[9] Cloninger CR. The Temperament and Character InventoryRevised. Centre for psychobiology of personality, Washington University, St Louis, Missouri. 1999.

[10] Corr PJ. J. A. Gray's reinforcement sensitivity theory: tests of the joint subsystem hypothesis of anxiety and impulsivity. Pers Individ Diff 2002;33:511-32.

[11] Corr PJ. Reinforcement sensitivity theory and personality. Neurosci Behav Rev 2004;28:317-32.

[12] Corr PJ, Kumari V, Wilson GD, Checkley S, Gray JA. Harm avoidance and affective modulation of the startle reflex: a replication. Pers Individ Diff 1997;22:591-3.

[13] Corr PJ, Wilson GD, Fotiadou M, Kumari V, Gray NS, Checkley $S$, et al. Personality and affective modulation of the startle reflex. Pers Individ Diff 1995;19:543-53.

[14] Costa PT, McCrae RR. Influence of extraversion and neuroticism on subjective well-being: happy and unhappy people. J Pers Soc Psychol 1980;38:668-78.

[15] De Pascalis V, Destro Fiore A, Sparita A. Personality, eventrelated potentials (ERP) and heart rate (HR): an investigation of Gray's theory. Pers Individ Diff 1996;20:733-46.

[16] De Pascalis V, Speranza O. Personality effects on attentional shift to emotional charged cues: ERP, behavioural and HR data. Pers Individ Diff 2000;29:217-38.

[17] De Pascalis V, Strippoli E, Riccardi P, Vergari F. Personality, event-related potential (ERP) and heart rate (HR) in emotional word processing. Pers Individ Diff 2004;36:873-91.

[18] Delplanque S, Lavoie ME, Hot P, Silvert L, Sequeira H. Modulation of cognitive processing by emotional valence studied through event-related potentials. Neurosci Lett 2004;356:1-4.

[19] Delplanque S, Silvert L, Hot P, Sequeira H. Event-related P3a and $\mathrm{P} 3 \mathrm{~b}$ in response to unpredictable emotional stimuli. Biol Psychol 2005;68:107-20.

[20] Eid M, Diener E. Intra-individual variability in affect: reliability, validity and personality correlates. J Pers Soc Psychol 1999;76:662-76.

[21] Fabiani M, Gratton G, Coles MGH. Event-related brain potentials: methods, theory and applications. In: Cacioppo JT, Tassinary LG, Bernston GG, editors. Handbook of psychophysiology. 2nd edn. New York: Cambridge university press; 2000. p. 53-84.

[22] Franken IHA, Muris P. Gray's impulsivity dimension: a distinction between reward sensitivity versus rash impulsiveness. Pers Individ Diff 2006;40:1337-47.

[23] Friedman D, Cycowicz YM, Gaeta H. The novelty P3: an eventrelated potential (ERP) sign of the brain's evaluation of novelty. Neurosci Behav Rev 2001;25:355-73.

[24] Gomez A, Gomez R. Personality traits of the behavioural approach and inhibition systems: associations with processing of emotional stimuli. Pers Individ Diff 2002;32: 1299-316.

[25] Gomez R, Cooper A, Gomez A. Susceptibility to positive and negative mood states: a test of Eysenck's. Gray's and Newman's theories. Pers Individ Diff 2000;29:351-65.

[26] Gomez R, Cooper A, McOrmond R, Tatlow S. Gray's reinforcement sensitivity theory: comparing the separable and joint subsystems hypotheses in the predictions of pleasant and unpleasant emotional information processing. Pers Individ Diff 2004;37:289-305.

[27] Gray JA, McNaughton N. The neuropsychology of anxiety: an inquiry into the functions of the septo-hippocampal system. 2nd ed. Oxford: Oxford University press; 2000.

[28] Gross JJ, Sutton SK, Ketelaar T. Relations between affect and personality: support for the affect-level and affectivereactivity views. Pers Soc Psychol Bull 1998;24:279-88.

[29] Hansenne M, Pinto E, Scantamburlo G, Renard B, Reggers J, Fuchs $S$, et al. Harm avoidance is related to mismatch negativity (MMN) amplitude in healthy subjects. Pers Individ Diff 2003;34:1039-48. 
[30] Heponiemi T, Keltikangas-Järvinen L, Puttonen S, Ravaja N. BIS/BAS sensitivity and self-rated affects during experimentally induced stress. Pers Individ Diff 2003;34:943-57.

[31] Hruby T, Marsalek P. Event-related potentials - the P3 wave. Acta Neurobiol Exp 2003;63:55-63.

[32] Keil A, Bradley MM, Hauk O, Rockstroh TE, Lang PJ. Large-scale neutral correlates of affective picture processing. Psychophysiology 2002;39:641-9.

[33] Lang PJ, Bradley MM, Cluthbert BN. International affective picture system (IAPS): instruction manual and affective ratings. Technical report A-4, The Center for Research in Psychophysiology, University of Florida. 1999.

[34] Öhman A. Fear and anxiety: evolutionary, cognitive and clinical perspectives. In: Lewis M, Haviland-Jones JM, editors. Handbook of emotions. N-Y: The Guilford press; 2000.

[35] Palomba D, Angrilli A, Mini A. Visual evoked potentials, heart rate responses and memory to emotional pictorial stimuli. Int J Psychophysiol 1997;27:55-67.

[36] Pélissolo A, Mallet L, Baleyete J-M, Michel G, Cloninger CR, Allilaire J-F, et al. The temperament and character inventoryrevised $(\mathrm{TCl}-\mathrm{R})$ : psychometric characteristics of the French version. Acta Psychiatr Scand 2005;112:126-33.

[37] Picton TW. The P300 wave of the human event-related potential. J Clin Neurophysiol 1992;9:456-79.

[38] Pizzagalli D, Regard M, Lehmann D. Rapid emotional face processing in the human right and left brain hemispheres: an ERP study. Neuroreport 1999;10:2691-8.

[39] Polich J. Theoretical overview of P3a and P3b. In: Polich J, editor. Detection of change: event-related potential and fMRI findings. Boston: Kluwer Academic Press; 2003. p. 83-98.
[40] Robinson MD. Running from William James' bear: a review of preattentive mechanisms and their contribution to emotional experience. Cogn Emotion 1998;12:667-96.

[41] Rossignol M, Anselme C, Vermeulen N, Philippot P, Campanella $S$. Categorical perception of anger and disgust facial expression is affected by non-clinical social anxiety: an ERP study. Brain Res 2007;1132:166-76.

[42] Rusting CL. Interactive effects of personality and mood on emotion-congruent memory and judgment. J Pers Soc Psychol 1999;77:1073-86.

[43] Smillie LD, Jackson CJ. Functional impulsivity and reinforcement sensitivity theory. J Pers 2006;74:47-83.

[44] Smillie LD, Jackson CJ, Dalgleish LI. Conceptual distinctions among Carver and White's (1994) BAS scales: a rewardreactivity versus trait impulsivity perspective. Pers Individ Diff 2006;40:1039-50.

[45] Smillie LD, Pickering AD, Jackson CJ. The new reinforcement sensitivity theory: implications for personality measurement. Pers Soc Psychol Rev 2006;10:320-35.

[46] Surakka V, Tenhunen-Eskelinen M, Hietanen JK, Sams M. Modulation of human auditory information processing by emotional visual stimuli. Cogn Brain Res 1998;7:159-63.

[47] Svrakic DMC, Przybeck TR, Cloninger CR. Mood states and personality traits. J Affect Disord 1992;24:217-26.

[48] Van de Velde M, Ghosh IR, Cluitmans PJ. Context related artefact detection in prolonged EEG recordings. Comput Methods Programs Biomed 1999;60:183-96.

[49] Verleger R. Event-related potentials and cognition: a critique of the context updating hypothesis and an alternative interpretation of P3. Behav Brain Sci 1988;11:343-56. 